\title{
Photophysical Effects behind the Efficiency of Hot Electron Injection in Plasmon-Assisted Catalysis: The Joint Role of Morphology and Composition
}

Yoel Negrín-Montecelo, ${ }^{1}$ Miguel Comesaña-Hermo, ${ }^{2, *}$ Larousse Khosravi Khorashad, ${ }^{3}$ Ana Sousa-Castillo, ${ }^{1}$ Zhiming Wang, ${ }^{4}$ Moisés Pérez-Lorenzo, ${ }^{1, *}$ Tim Liedl, ${ }^{5}$ Alexander O. Govorov, ${ }^{3,4, *}$ Miguel A. Correa-Duarte ${ }^{1, *}$

${ }^{1}$ Department of Physical Chemistry, Singular Center for Biomedical Research (CINBIO), Southern Galicia Institute of Health Research (IISGS), and Biomedical Research Networking Center for Mental Health (CIBERSAM), Universidade de Vigo, 36310 Vigo, Spain

${ }^{2}$ Université de Paris, ITODYS, CNRS, UMR 7086, 15 rue J-A de Baïf, F-75013 Paris, France

${ }^{3}$ Department of Physics and Astronomy, Ohio University, Athens, Ohio 45701, United States

${ }^{4}$ Institute of Fundamental and Frontier Sciences, University of Electronic Science and Technology of China, Chengdu, 610054, China

${ }^{5}$ Fakultät für Physik and Center for Nanoscience, Ludwig-Maximilians-Universität, Geschwister-Scholl-Platz 1, 80539 München, Germany

\section{Experimental methods}

Materials: Tetrachloroauric acid $\left(\mathrm{HAuCl}_{4} \cdot 3 \mathrm{H}_{2} \mathrm{O}\right)$, hexadecyltrimethylammonium bromide (CTAB), sodium borohydride $\left(\mathrm{NaBH}_{4}\right)$, silver nitrate $\left(\mathrm{AgNO}_{3}\right)$, hydrochloric acid $(\mathrm{HCl})$, L-ascorbic acid (AA), poly(allylamine hydrochloride) (PAH), sodium chloride ( $\mathrm{NaCl})$, poly(styrenesulfonate) (PSS), tetraethylorthosilicate (TEOS), ammonium hydroxide solution $28-30 \%\left(\mathrm{NH}_{4} \mathrm{OH}\right)$, sodium citrate $\left(\mathrm{Na}_{3} \mathrm{C}_{6} \mathrm{H}_{5} \mathrm{O}_{7}\right)$, sodium formate and formic acid, were purchased from Sigma-Aldrich. $\mathrm{TiO}_{2}$ nanoparticles of $5 \mathrm{~nm}$ were purchased from Nanoamor. Pure grade ethanol and Milli-Q grade water were used in all preparations.

Synthesis of Au nanorods with the longitudinal LSPR centered at $640 \mathrm{~nm}$ and PSS Coating: AuNRs with a length of $57( \pm 7) \mathrm{nm}$ and width of $27( \pm 3) \mathrm{nm}$ are synthesized with a modification of the protocol described by Scarabelli et all. ${ }^{[1]}$ In order to tune the longitudinal LSPR maximum to $640 \mathrm{~nm}$, some modifications have been made: $2 / 3$ and 1/4 of the original amounts of $\mathrm{AgNO}_{3}$ and seeds solutions in the growth process have been used, respectively. The AuNRs are subsequently coated with a layer of a negatively charged polyelectrolyte (PSS). ${ }^{[2]}$

Synthesis of Au@Ag nanorods and PSS Coating: Au@Ag NRs with a length of $61( \pm 8)$ nm and width of $26( \pm 2) \mathrm{nm}$ are synthesized following a previously reported procedure. ${ }^{[3]} \mathrm{In}$ this manner, the longitudinal LSPR maximum is centered at $640 \mathrm{~nm}$. The Au@AgNRs are subsequently coated with a layer of a negatively charged polyelectrolyte (PSS).

Synthesis and fuctionalization of silica beads: Monodisperse silica spheres with a diameter of $500 \mathrm{~nm}$ are prepared using a modified Stöber method. ${ }^{[4]}$ Typically, an ethanolic TEOS solution $(1.7 \mathrm{~mL}, 1.2 \mathrm{M})$ is added to a solution containing ethanol $(18.12 \mathrm{~mL})$, ammonium hydroxide $(1.96 \mathrm{~mL})$, and water $(3.21 \mathrm{~mL})$. This mixture is stirred at room temperature for $2 \mathrm{~h}$. The excess of reagents is removed by three centrifugationredispersion cycles with ethanol $(5000 \mathrm{rpm}, 30 \mathrm{~min})$. Subsequently, $\mathrm{PAH}$ is dissolved in an aqueous $\mathrm{NaCl}$ solution $(0.5 \mathrm{M}$ and $\mathrm{pH}=5.0)$ with a final polymer concentration of $1 \mathrm{mg} / \mathrm{mL}$. Then, $30 \mathrm{~mL}$ of the positively charged PAH solution are added to the silica nanoparticles $(30 \mathrm{mg})$ and stirred at room temperature for 30 $\mathrm{min}$. The excess of reagents is removed by three centrifugation-redispersion cycles with water $(5000 \mathrm{rpm}, 30$ $\mathrm{min}$ ) and the solid is redispersed in $30 \mathrm{~mL}$ of Milli-Q water. 
Deposition of $\mathrm{TiO}_{2}: 50 \mathrm{mg}$ of $\mathrm{TiO}_{2}(5 \mathrm{~nm})$ are dispersed in $100 \mathrm{~mL}$ of a sodium citrate solution $(2.5 \mathrm{mM})$ and sonicated for $1 \mathrm{~h}$ with an ultrasonic tip. The aggregated particles of $\mathrm{TiO}_{2}$ are removed by centrifugation (3500 rpm, $10 \mathrm{~min}$ ). Then, $30 \mathrm{~mL}$ of the $\mathrm{SiO}_{2} @ \mathrm{PAH}$ are added to $18 \mathrm{~mL}$ of the solution of $\mathrm{TiO}_{2}$ and stirred at room temperature for $60 \mathrm{~min}$. The excess of $\mathrm{TiO}_{2}$ is removed by three centrifugation-redispersion cycles $(6000 \mathrm{rpm}$, $30 \mathrm{~min}$ ). Finally, the product is redispersed in $30 \mathrm{~mL}$ of water.

Assembly of PSS functionalized Au NRs and $\mathrm{Au} @ \mathrm{Ag}$ NRs onto $\mathrm{SiO}_{2} @ \mathrm{TiO}_{2}$ functionalized with PAH: 0.25, 0.5 and $1 \mathrm{~mL}$ of the solution of NRs@PSS $(0.5 \mathrm{mM})$ were added to $5 \mathrm{mg}$ of functionalized silica nanoparticles in each case. The mixtures are stirred at room temperature for $3 \mathrm{~h}$ and washed by three centrifugationredispersion cycles with water $(6000 \mathrm{rpm}, 30 \mathrm{~min})$. The resulting product is redispersed in $5 \mathrm{~mL}$ of water. The metal $/ \mathrm{TiO}_{2}$ molar ratio for both plasmonic objects are $0.05,0.1$ and 0.2 , as calculated by ICP.

Photocatalytic degradation of rhodamine B: The photocatalytic activity of the $\mathrm{SiO}_{2} @ \mathrm{TiO}_{2} @ \mathrm{NPs}_{2}$ nanohybrids is evaluated by the degradation of rhodamine $\mathrm{B}(\mathrm{RhB})$ in an aqueous solution inside a controlled water bath at $25{ }^{\circ} \mathrm{C}$ under light irradiation from a $300 \mathrm{~W}$ Xe lamp in a LOT solar simulator $(\lambda=350-2400 \mathrm{~nm})$. Reference experiments have been performed using different filters that block specific ranges of the solar spectrum. These filters induce a decrease in the transmitted light of $\sim 10 \%$. The solutions are prepared by mixing $200 \mu \mathrm{L}$ of an aqueous solution of $\mathrm{RhB}(1 \mathrm{mM}), 4 \mathrm{~mL}$ of the hybrid solution $(1 \mathrm{mg} / \mathrm{mL})$ and $15.8 \mathrm{~mL}$ of Milli-Q water. The mixtures are stirred for $1 \mathrm{~h}$ in the dark to blend well and allow the adsorption-desorption equilibrium to take place before irradiation. Aliquots of $2.5 \mathrm{~mL}$ are taken within $30 \mathrm{~min}$ intervals during the experiments in order to measure the variation in the absorbance of the dye.

Photocatalytic hydrogen generation assisted by formic acid: The hydrogen production from a FA-SF solution is carried out in a $3 \mathrm{~mL}$ flask. Typically, $2.5 \mathrm{~mL}$ of a catalyst dispersion in water $\left(0.1 \mathrm{mg}\right.$ of $\left.\mathrm{TiO}_{2}\right)$ is mixed with $0.108 \mathrm{~g}$ of sodium formate and $20 \mu \mathrm{L}$ of formic acid. The gases are purged for 2 min and the flask is sealed. The dispersion is magnetically stirred inside a water bath at $35{ }^{\circ} \mathrm{C}$ under light irradiation with the solar simulator $(\lambda=350-2400 \mathrm{~nm}$ ). After $1 \mathrm{~h}$, the gases are analyzed with a gas chromatographer (Agilent Technologies 7829A GC system) in order to measure the volume of $\mathrm{H}_{2}$ generated.

\section{Theoretical modelling}

For the theoretical modeling of the as-synthesized Au and Au@Ag NRs, we used COMSOL software. Electromagnetic wave is incident on the NRs which are randomly oriented in solution. Therefore, in the simulation, one needs to consider three orthogonal wave propagation directions incident on the structures. Averaging over three orthogonal directions will ensure decent approximation of the absorption $\left(\sigma_{a b s}\right)$, scattering $\left(\sigma_{\text {scat }}\right)$, and extinction $\left(\sigma_{\text {ext }}\right)$ cross section spectra where $\sigma_{\text {ext }}=\sigma_{a b s}+\sigma_{\text {scat }}$. Due to the symmetry of the NRs with respect to the polarization field $(E)$ of the light, the averaged cross section spectra read:

$$
\sigma_{\text {average }}=\frac{\sigma_{k\|x, E\| y}+\sigma_{k\|x, E\| \mid z}+\sigma_{k\|y, E\| x}+\sigma_{k\|y, E\| z}+\sigma_{k\|z, E\| x}+\sigma_{k\|z, E\| y}}{6}
$$

Another important concept to consider in the model is inhomogeneous broadening of the empirical dielectric functions of gold and silver which occurs due to polydispersity of the metallic composition. The broadening of the extinction spectra of $\mathrm{Au}$ and $\mathrm{Au} @ \mathrm{Ag}$ NRs is implied by the experimental measurement in Figure 1g. To account for this broadening, a previously published procedure is used, ${ }^{[5]}$ in which the dielectric function of the metal reads as,

$$
\varepsilon_{\text {metal }}(\omega)=\varepsilon_{\text {bulk }}(\omega)+\frac{\omega_{p}^{2}}{\omega^{2}+i \omega \cdot \Gamma_{\text {bulk }, D}}-\frac{\omega_{p}^{2}}{\omega^{2}+i \omega \cdot \Gamma_{\text {bulk,broadened }}} .
$$

Here, $\varepsilon_{b u l k}(\omega)$ is the frequency dependent empirical dielectric function of $\mathrm{Au}(\mathrm{Ag}){ }^{[6]}$ The Drude plasma frequency and Drude damping are taken as $\omega_{p, A u}=8.9 \mathrm{eV}, \omega_{p, A g}=9.3 \mathrm{eV}, \Gamma_{b u l k, D, A u}=$ 
$0.076 \mathrm{eV}$ and $\Gamma_{b u l k, D, A g}=0.02 \mathrm{eV}$. The additional damping factor to include inhomogeneity is taken as $\Gamma_{\text {bulk,broadened }}=3 \cdot \Gamma_{\text {bulk,D }}$ for both gold and silver. Figure $1 \mathrm{~h}$ shows the calculated cross sections for the two structures of $\mathrm{Au}$ and $\mathrm{Au} @ \mathrm{Ag}$ NRs averaged over three orthogonal directions of electric field polarization. Moreover, Figure 1i, j depicts the geometrical parameters of the monometallic and bimetallic NRs. In this manner, both longitudinal and transverse plasmon modes are seen in Figure $1 \mathrm{k}$.

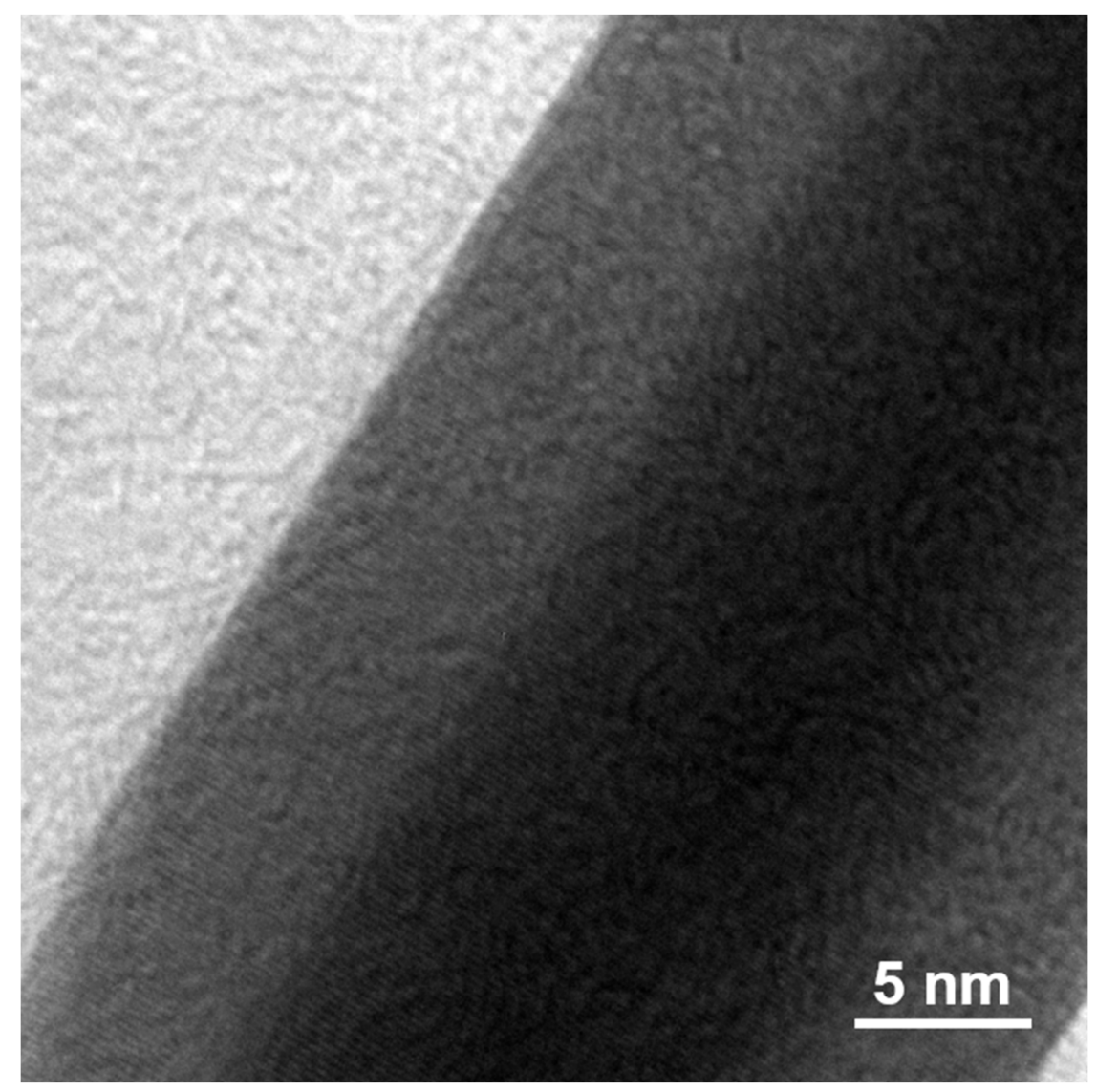

Figure S1. HRTEM image of a Au@Ag NR in which the core@shell structure can be observed.
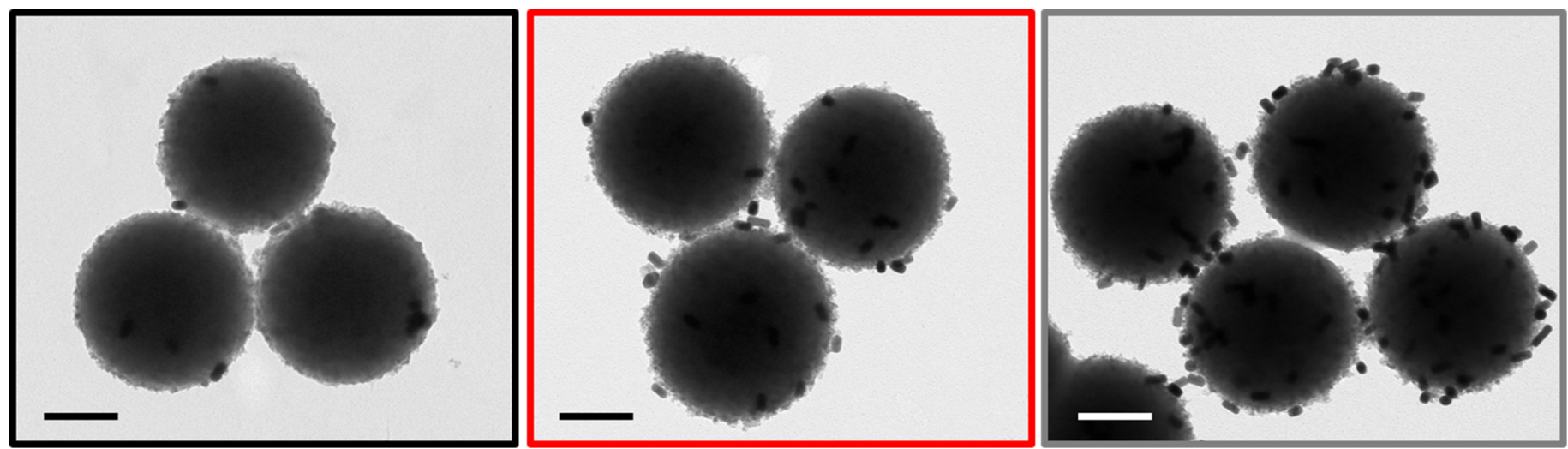

Figure S2. TEM images of the hybrids composed with $\mathrm{Au}$ NRs and metal/ $\mathrm{TiO}_{2}$ aspect ratios of: 0.05 (black), 0.1 (red) and 0.2 (grey). Scale bars represent $200 \mathrm{~nm}$. 

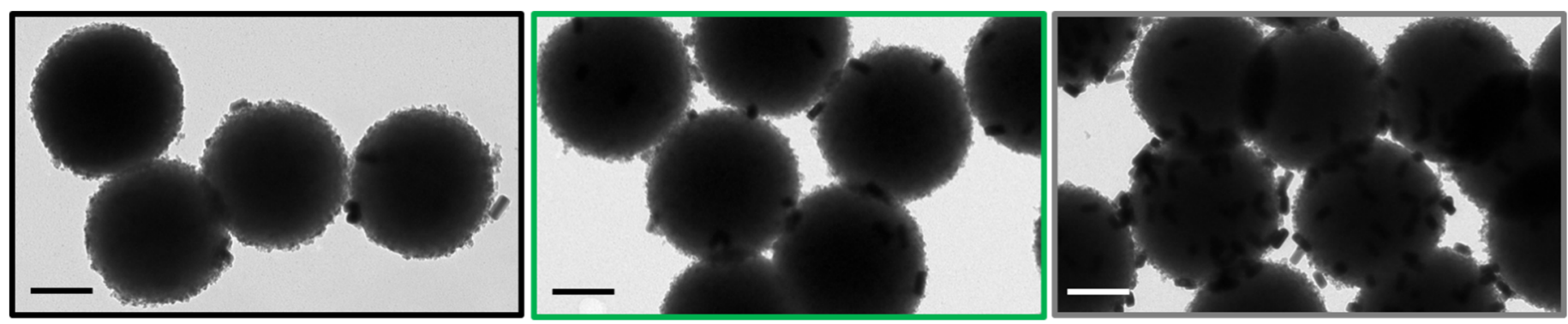

Figure S3. TEM images of the hybrids composed with $\mathrm{Au} @ \mathrm{Ag} \mathrm{NRs}$ and metal/ $/ \mathrm{TiO}_{2}$ aspect ratios of: 0.05 (black), 0.1 (green) and 0.2 (grey). Scale bars represent $200 \mathrm{~nm}$.

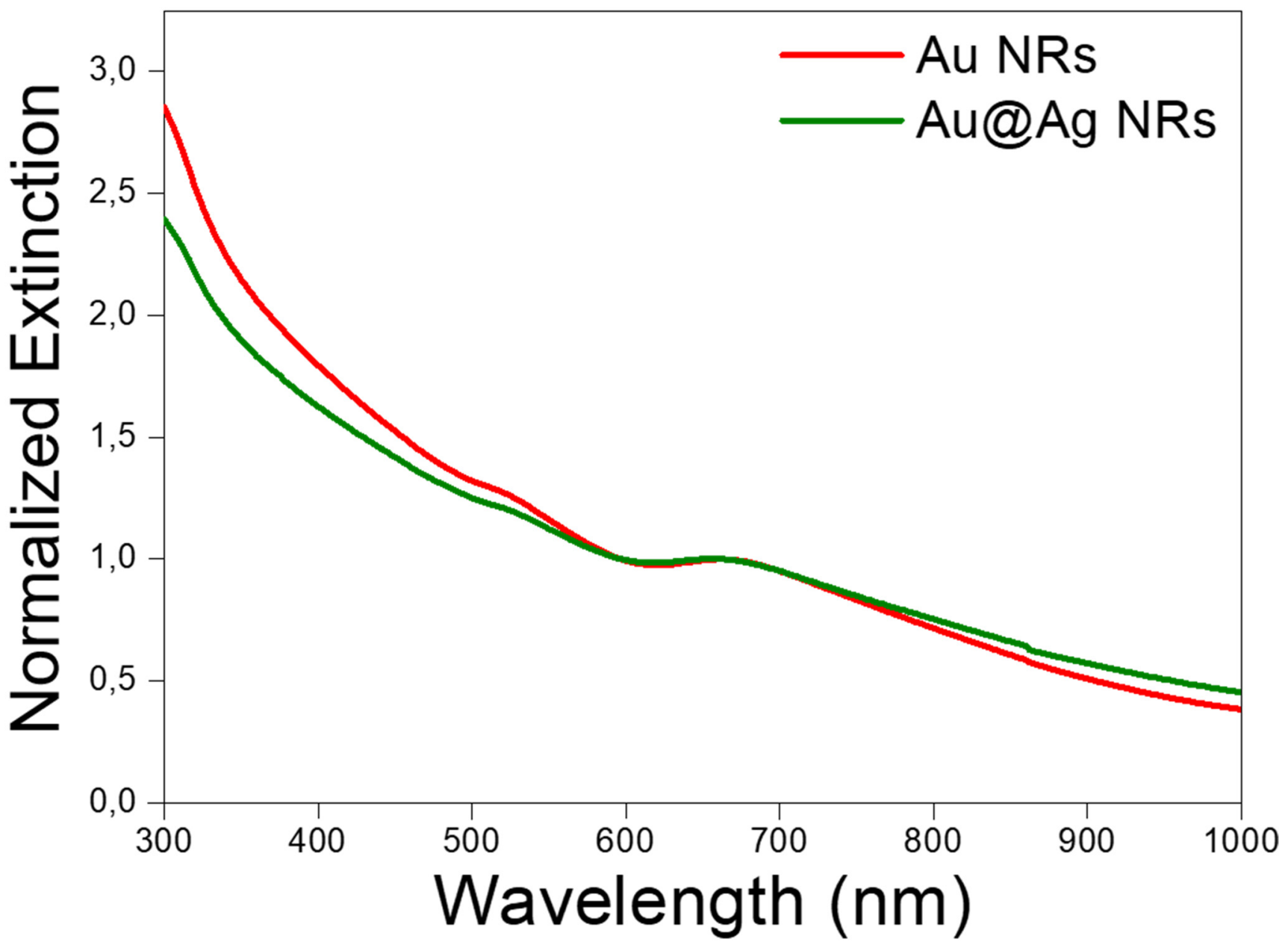

Figure S4. Extinction spectra of the $\mathrm{SiO}_{2} @ \mathrm{TiO}_{2} @$ metal nanohybrids synthesized with Au NRs (red line) or Au@Ag NRs (green line). The spectra have been normalized to the maximum of the longitudinal signature of the plasmonic NRs. 


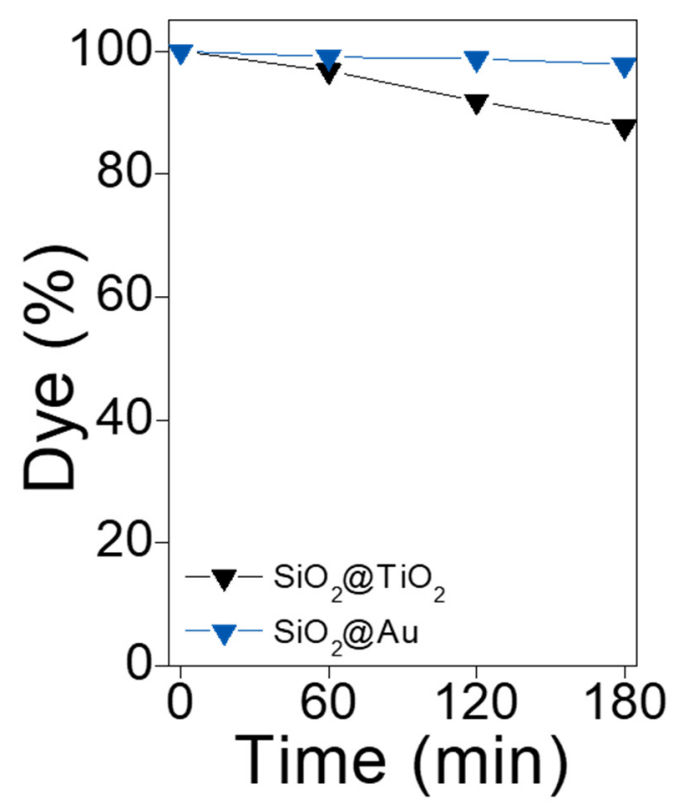

Figure S5. Photocatalytic activity profiles for the degradation of RhB with the reference samples: silica substrates functionalized either with $\mathrm{TiO}_{2}$ NPs (black triangles) or with Au NRs (blue triangles).

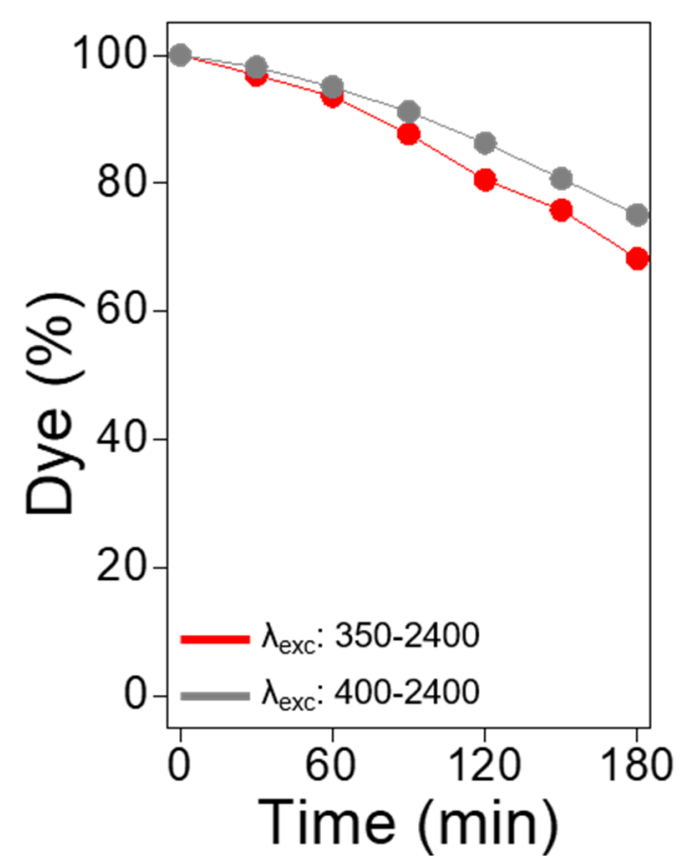

Figure S6. Photocatalytic activity profiles for the degradation of RhB with the hybrids formed with Au NRs (ideal $\mathrm{Au} / \mathrm{TiO}_{2}$ molar ratio of 0.1 ) with different irradiation wavelengths. 

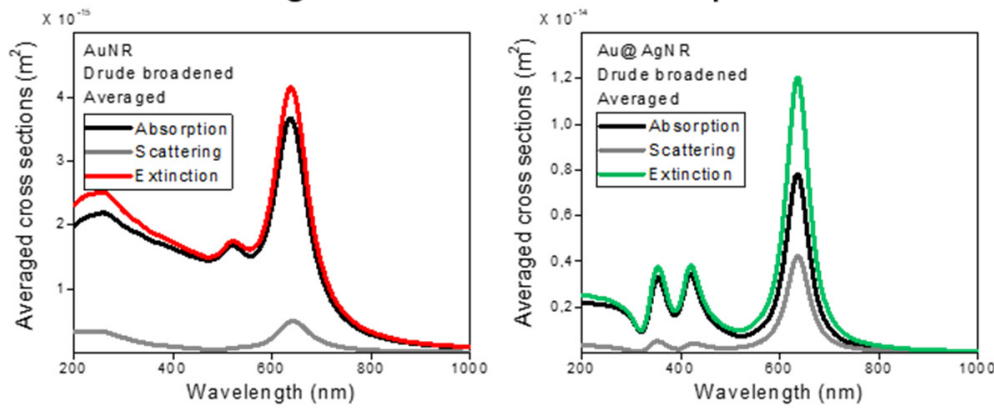

Spectra for polarized excitation
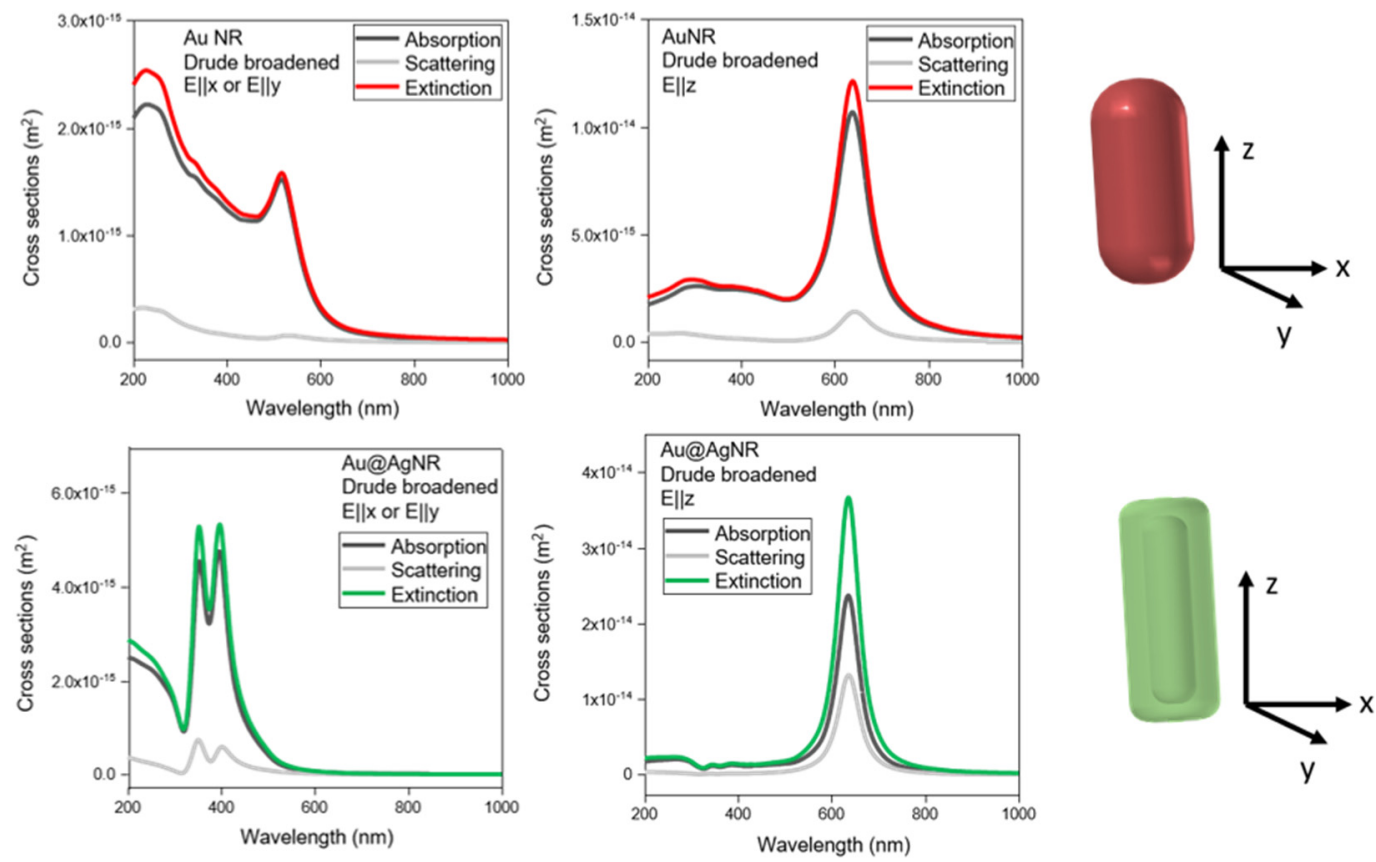

Figure S7. Unpolarized and polarized calculated spectra for the Au (red) and Au@Ag (green) NRs. These spectra reveal the origin of the plasmon modes and the nature of the extinction signals.

\section{References}

1. Scarabelli, L.; Sánchez-Iglesias, A.; Pérez-Juste, J.; Liz-Marzán, L.M. A “Tips and Tricks” Practical Guide to the Synthesis of Gold Nanorods. J. Phys. Chem. Lett. 2015, 6, 4270-4279.

2. Sousa-Castillo, A.; Comesaña-Hermo, M.; Rodríguez-González, B.; Pérez-Lorenzo, M.; Wang, Z.; Kong, X.-T.; Govorov, A.O.; Correa-Duarte, M.A. Boosting Hot Electron-Driven Photocatalysis through Anisotropic Plasmonic Nanoparticles with Hot Spots in Au-TiO 2 Nanoarchitectures. J. Phys. Chem. C 2016, 120, 11690-11699.

3. Gómez-Graña, S.; Pérez-Juste, J.; Alvarez-Puebla, R.A.; Guerrero-Martínez, A.; Liz-Marzán, L.M. Self-Assembly of Au@Ag Nanorods Mediated by Gemini Surfactants for Highly Efficient SERSActive Supercrystals. Adv. Opt. Mater. 2013, 1, 477-481.

4. Stöber, W.; Fink, A.; Bohn, E. Controlled growth of monodisperse silica spheres in the micron size range. J. Colloid Interface Sci. 1968, 26, 62-69.

5. Kuzyk, A.; Schreiber, R.; Zhang, H.; Govorov, A.O.; Liedl, T.; Liu, N. Reconfigurable 3D plasmonic metamolecules. Nat. Mater. 2014, 13, 862-866.

6. Johnson, P.B.; Christy, R.W. Optical Constants of the Noble Metals. Phys. Rev. B 1972, 6, 4370-4379. 